\title{
Highest Energy Astrophysical Photons Detected
}

\section{An experiment operating at high altitudes in Tibet has detected the highest energy photons ever observed from an astrophysical source, the Crab Nebula.}

\section{by Rene A. Ong*}

$\mathrm{V}$ ery-high-energy (VHE) gamma-ray astronomy gives the view of the Universe at photon energies above $100 \mathrm{GeV}\left(10^{11} \mathrm{eV}\right)$. To produce photons at these high energies requires a cosmic accelerator many times more powerful than the Large Hadron Collider. Known gamma-ray sources, such as pulsars

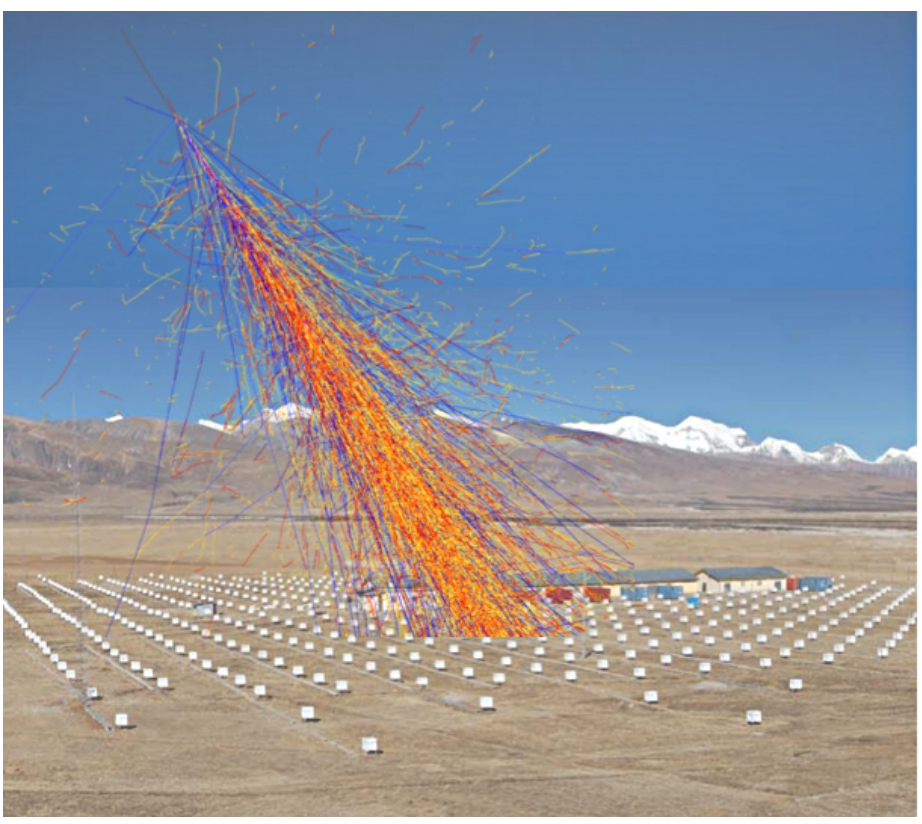

Figure 1: The Tibet $\mathrm{AB} \gamma$ experiment is located at an altitude of $4300 \mathrm{~m}$ in Yangbajing, China. With an array of scintillation detectors and underwater water-Cherenkov detectors covering $65,700 \mathrm{~m}^{2}$, the experiment measures the secondary particles that are produced when a high-energy photon strikes the upper atmosphere. An artist's depiction of such an air shower is shown in the image. (Tibet AS $\gamma$ Collaboration)

\footnotetext{
*Department of Physics and Astronomy, University of California, Los Angeles, CA, USA
}

and active galactic nuclei, harbor intense electromagnetic or gravitational fields for accelerating particles that subsequently produce VHE photons. Astrophysicists have models for how this acceleration occurs, but uncertainties remain over such issues as the maximum particle energy achievable through these mechanisms. To help address these questions, researchers search for the highest energy-and rarest-gamma rays using ground-based experiments with very large collection areas. Now, for the first time, such an experiment has detected photons above $100 \mathrm{TeV}\left(10^{14} \mathrm{eV}\right)$ from an astrophysical source, namely, the Crab Nebula [1]. Not only does this detection open up a new view of the Universe, it also confirms the standard theoretical framework for particle acceleration and radiation in one of the beststudied astrophysical sources.

When a VHE particle-either gamma ray or cosmic ray-reaches us from the cosmos, it interacts with the Earth's atmosphere to create an extensive air shower of secondary particles and photons that propagate down to the surface of the Earth (Fig. 1). By measuring these secondary products from the ground, we can gather information on the primary VHE particle [2]. One of the main motivations of studying gamma rays in this way has been to pinpoint astrophysical sites of extreme particle acceleration that could also explain the origin of cosmic rays. Some of the first projects to perform such measurements were air shower arrays, which detect the particles in the shower via a widely distributed array of detectors, typically either scintillator or water-Cherenkov detectors. By measuring the densities and arrival times of secondary particles at numerous locations on the ground, air shower arrays estimate the energy and arrival direction of the incoming primary particle, as well as determine whether it was a gamma-ray photon or a cosmic ray.

The first definitive detection of a VHE gamma-ray source, the Crab Nebula, was made by the Whipple $10 \mathrm{~m}$ Telescope in 1989 [3]. Whipple was not an air shower array but rather an atmospheric Cherenkov telescope, which captures secondary photons (as opposed to secondary particles) using dish-shaped reflectors. Although the Whipple detection of $1-\mathrm{TeV}$ photons was a landmark event, the fact that the Crab was the first source to be detected was not sur- 
prising. The Crab, the remnant of a supernova explosion observed on Earth in $1054 \mathrm{CE}$, is perhaps the most studied source in astronomy and has very luminous emission over all wavelengths. In the VHE band, the Crab's emission is bright and steady, powered most likely by a wind of relativistic electrons blowing off the Crab Pulsar. This wind model-supported by the shape of the VHE spectrum-assumes that the electrons interact with low-energy ambient photons through inverse-Compton scattering, producing gamma-ray photons [4].

Motivated by the Whipple detection and hoping to see higher-energy photons, a collaboration of Japanese and Chinese scientists constructed the Tibet air shower experiment (Tibet AS $\gamma$ ) in 1990. This array, which initially consisted of 49 scintillation detectors covering an area of $11,000 \mathrm{~m}^{2}$, benefits from a high altitude of $4300 \mathrm{~m}$, which allows it to detect a wider range of gamma-ray energies than the first generation of air shower arrays [5]. In 1999, Tibet AS $\gamma$ made the first detection of the Crab Nebula by an air shower array [6]. The experiment has been periodically upgraded with additional scintillation detectors (now totaling around 600) and, more recently, with the installation of 24 underground water-Cherenkov detectors, which measure muons. Since gamma-ray initiated air showers are expected to have far fewer muons than those initiated by cosmic rays, the measurement of the muon content rejects the background events due to cosmic rays, focusing on those due to gamma-ray photons. Thanks to this additional equipment, the Tibet AS $\gamma$ has significantly improved sensitivity at both low $(\sim 1 \mathrm{TeV})$ and high $(\sim 100 \mathrm{TeV})$ energies.

Over the last 30 years, the Tibet AS $\gamma$ has been accompanied by atmospheric Cherenkov telescopes, such as H.E.S.S., MAGIC, and VERITAS and by array-type experiments like the Milagro experiment. These various ground-based observatories have revolutionized our understanding of the VHE universe by discovering and characterizing close to 200 sources of $\mathrm{TeV}$ gamma radiation [7]. However, none of these sources was detected at $100-\mathrm{TeV}$ energies.

That situation has now changed. The Tibet AS $\gamma$ collaboration reports the detection of a clear gamma-ray signal from the direction of the Crab Nebula at energies above $100 \mathrm{TeV}$ [1]. The calculated photon spectrum shows good agreement with previous measurements at lower energies and a smooth continuation of the inverse-Compton model to the highest energies [4]. It's worth noting that a similar result has recently been reported at conferences by the successor to Milagro: the High-Altitude Water Cherenkov (HAWC) Observatory, which started regular operations in 2014. HAWC has a high-altitude $(4100 \mathrm{~m})$ location, large muon detectors, and improved reconstruction capabilities that-like Tibet $\mathrm{AS} \gamma$-give it a superior performance compared to earlier air shower arrays. The HAWC Collaboration recently submitted their $100-\mathrm{TeV}$ observations of the Crab for publication [8].
The work reported here represents the achievement of a long-held goal to measure $100-\mathrm{TeV}$ gamma rays. Although there is nothing magic about $100 \mathrm{TeV}$, the detections by Tibet AS $\gamma$ and HAWC confirm that our standard picture of particle acceleration and radiation in the Crab Nebula continues to higher energies in a smooth fashion. This, in turn, argues that the primary electrons being accelerated in the Crab approach energies of $1 \mathrm{PeV}\left(10^{15} \mathrm{eV}\right)$.

Looking toward the future, extending the Crab spectrum to increasingly higher energies would be important to ensure that no new component appears. Detection of other objects above $100 \mathrm{TeV}$ can be expected although-because of absorption by intergalactic radiation fields-only sources in our Galaxy or its neighborhood are visible at these energies. Nonetheless, HAWC has shown that there are many Galactic sources in the TeV band [9], and hence future detections could shed light on the highest energy accelerators in the Milky Way, the so-called PeVatrons that are needed to explain what powers the peta-electronvolt cosmic rays that get to Earth. Similarly, if photons at these highest energies can be correlated with neutrino events detected by the IceCube experiment at the South Pole, it would provide important clues about the origin of the highest energy neutrinos.

The many recent discoveries in the field of VHE gammaray astronomy have motivated the development of new, more sensitive instruments to probe the $100-\mathrm{TeV}$ frontier. One example is the Large High Altitude Air Shower Observatory (LHAASO), which is a new air shower experiment that was recently inaugurated in Sichuan, China. Additionally, development continues on the Cherenkov Telescope Array (CTA), which will comprise two large arrays of atmospheric Cherenkov telescopes at sites in Chile and Spain. There also exists significant interest in a southern hemisphere high-altitude air shower experiment [10].

This research is published in Physical Review Letters and on the arXiv.

\section{REFERENCES}

[1] M. Amenomori et al. (Tibet AS $\gamma$ Collaboration), "First detection of photons with energy beyond $100 \mathrm{TeV}$ from an astrophysical source," Phys. Rev. Lett. 123, 051101 (2019).

[2] P. Morrison et al., "On gamma-ray astronomy," Nuovo Cimento 7, 858 (1958); G. Cocconi, in Proceedings of the Moscow Cosmic Ray Conference, edited by G. B. Zdanovic and V. J. Zatsepin (Int. Union, Moscow, 1960), Vol. 2, p. 309.

[3] T. C. Weekes et al., "Observation of TeV gamma rays from the Crab nebula using the atmospheric Cerenkov imaging technique," Astrophys. J. 342, 379 (1989).

[4] O. C. de Jager and A. K. Harding, "The expected high-energy to ultra-high-energy gamma-ray spectrum of the Crab Nebula," Astrophys. J. 396, 161 (1992); A. M. Atoyan and F. A. Aharonian, "On the mechanisms of gamma radiation in the Crab Nebula," Mon. Not. R. Astron. Soc. 278, 525 (1996). 
[5] For a review of the field of VHE gamma-ray astronomy before 2000 , see R. A. Ong, "Very high-energy gamma-ray astronomy," Phys. Rep. 305, 93 (1998).

[6] M. Amenomori et al. (Tibet AS $\gamma$ Collaboration), "Observation of Multi-TeV Gamma Rays from the Crab Nebula using the Tibet Air Shower Array," Astrophys. J. 525, L93 (1999).

[7] See http://tevcat.uchicago.edu/.

[8] A. U. Abeysekara et al. (HAWC Collaboration), "Measurement of the Crab Nebula at the highest energies with HAWC,"
arXiv:1905.12518.

[9] A. U. Abeysekara et al., "The 2HWC HAWC Observatory gamma-ray catalog," Astrophys. J. 843, 40 (2017).

[10] LHAASO: http://english.ihep.cas.cn/lhaaso/; CTA: https://www .cta-observatory.org/; southern hemisphere air shower experiment: https://arxiv.org/abs/1902.08429.

10.1103/Physics.12.87 